\title{
Efficient Proxy Re-encryption with Private Keyword Searching in Untrusted Storage
}

\author{
Xi Chen \\ Key Laboratory of Communication \& Information Systems (Beijing Jiaotong University), \\ Beijing Municipal Commission of Education, Beijing 100044, China \\ Email: 09120113@bjtu.edu.cn \\ Yong Li \\ Key Laboratory of Communication \& Information Systems (Beijing Jiaotong University), \\ Beijing Municipal Commission of Education, Beijing 100044, China \\ Email: li.yong9@gmail.com
}

\begin{abstract}
Cloud computing is an important trend that in many ways is beginning to fulfill the early promise of the Internet and creating unanticipated change in computing paradigm. As promising as cloud computing is, this paradigm brings forth new security and privacy challenges when operating in the untrusted cloud scenarios. Motivated by the challenging problem "Private Searching over Encrypted Data”, we propose a new cryptographic primitive, Proxy Re-encryption with Private Searching (PRPS for short). The PRPS scheme enables the data users and owners efficiently query and access files stored in untrusted cloud, while keeping query privacy and data privacy from the cloud providers. The concrete construction is based on proxy re-encryption, public key encryption with keyword search and the dual receiver cryptosystem. Extensive analysis shows that our scheme is efficient and semantically secure under the BDH assumption.
\end{abstract}

Index Terms - public key encryption with keyword search; proxy re-encryption; untrusted cloud; private searching

\section{INTRODUCTION}

Cloud computing is an important trend which is beginning to fulfill the early promise of the Internet and creating unanticipated change in computing paradigm. However, a significant barrier to the adoption of cloud computing is that data owners fear of confidential data leakage and lose of privacy in the cloud [1]. These concerns originate from the fact that cloud providers are usually operated by commercial providers which are very likely to be outside of the trusted domain of the users. Data confidentialty against cloud providers is hence frequently desired when users outsource data for storage in the cloud [2].

Our work is motivated by the following scenario. Data owners, cloud storage providers and data users are separated geographically. A data owner stores his files in an encrypted form in the untrusted cloud, and retrieves them wherever and whenever he wants. What's more, he wants to share his files with other data users. The user sends a query for files containing certain keywords to the cloud provider. The desired requirements are: 1 ) The user can decrypt the files uploaded by the data owner with his private key; 2) The cloud provider can search whether the encrypted files contain some keywords; 3) The cloud provider ought to keep blind to the files content and the query keywords of the user; 4) The user could finish query and decryption with a thin client which demands computing overhead as small as possible. We call such kind of problem as "Private Searching on Encrypted Data” (PSED for short).

\section{A. Related work}

Proxy Re-Encryption (PRE). PRE is a cryptographic primitive, where a (potentially untrusted) proxy is given a re-encryption key $r k_{1 \rightarrow 2}$ that allows it to translate a message $m$ encrypted under public key $p k_{1}$ into a cipher texts under a public key $p k_{2}$, without being able to see anything about the encrypted messages. In [3], Ateniese et al. proposed a single-use, unidirectional, but not transparent Proxy Re-Encryption schemes based on bilinear maps.

Public key encryption with keyword search (PEKS). In PEKS scheme, Alice creates a trapdoor with her private key and a keyword, and sends it to S. S uses a test algorithm with inputting encrypted keyword, trapdoor and user's public key. If matches, it outputs 1 and 0 otherwise. PEKS supports that a user could search for some files containing certain keywords in untrusted storage servers, and at the same time, the servers keep blind to the privacy of file and the keyword. In [4], Boneh et al. proposed a public key encryption with keyword search scheme.

Dual receiver cryptosystem. Diament et al [5] first introduced the notion of an efficient dual receiver cryptosystem, which enables a cipher text to be decrypted by two independent receivers. The main disadvantage of the dual receiver cryptosystem is that the server needs to send an auxiliary private key to a client for decrypting a partial cipher text, which is insecure in the real environment [6].

Liu et al. [6] improved the PEKS by inspiring the idea of dual receiver cryptosystem, and proposed an efficient 
privacy preserving keyword search scheme. However, this scheme exists an inherent problem. It is one specific case applicable in the setting that the data owner and data user is the same one. Shao et al. [7] introduced the concept of proxy re-encryption with keyword search (PRES), in particular the concept of bidirectional PRES, against the chosen cipher text attack. Their scheme is based on the techniques for PRE in [8] and the IBE schemes in [9]. Note that the third party is trusted and this scheme improved the security level with the sacrifice of efficiency.

Note that there are further related work [10][11] and the latest work in Structured Encryption [12], which also considered the problem of private querying on encrypted data, i.e. enabling user efficiently query and retrieve the encrypted files containing specific keywords.

\section{B. Our contributions}

Main contributions of this paper can be summarized as follows.

1) We proposed a new cryptographic primitive, Proxy Re-encryption with Private Searching (PRPS), and the new PRPS construction combines technologies from PRE, PEKS and dual receiver cryptosystem. The PRPS scheme is able to protect the data privacy and the users' queries privacy simultaneously during the search process. And it is provably secure under the $\mathrm{BDH}$ assumption in random oracle model.

2) The PRPS scheme enables the decrease of computing overhead for the user.

3) It reduces the modification of encrypted sharing file storage when different users accessing the cloud provider.

The rest of this paper is organized as follows. Section II discusses some preliminaries. Section III provides the Proxy Re-encryption with Private Searching model and its security definition. Section IV introduces the construction for PRPS. In Section V, we analyze the PRPS scheme in terms of its security and efficiency. We conclude this paper in Section VI.

\section{PRELIMINARIES}

Let $G_{1}$ and $G_{2}$ be two cyclic groups of some large prime order $q$. We view $G_{1}$ as an additive group and $G_{2}$ as a multiplicative group.

Definition 2.1 (Bilinear Maps): We call $e$ a bilinear map if $e: G_{1} \times G_{1} \rightarrow G_{2}$ is a map with the following properties:

1) Computable: given $g, h \in G_{1}$, there is a polynomial time algorithms to compute $e(g, h) \in G_{2}$.

2) Bilinear: for any integers $x, y \in[1, q]$, we have $e\left(g^{x}, g^{y}\right)=e(g, g)^{x y}$.

3) Non-degenerate: if $g$ is a generator of $G_{1}$, then $e(g, g)$ is a generator of $G_{2}$.
Definition 2.2 (BDH Parameter Generator): We say that a randomized algorithm $I G$ is a $\mathrm{BDH}$ parameter generator if $I G$ takes a sufficiently large security parameter $K>0$, runs in polynomial time in $K$, and outputs the description of two groups $G_{1}$ and $G_{2}$ of the same prime order $q$ and the description of a bilinear map $e: G_{1} \times G_{1} \rightarrow G_{2}$

Definition 2.3 (BDH Problem): Given a random element $g \in G_{1}$, as well as $g^{x}, g^{y}$ and $g^{z}$, for some $^{x, y, z \in Z_{q}^{*}}$, compute $e(g, g)^{x y z} \in G_{2}$.

Definition 2.4 (BDH Assumption): If $I G$ is a $\mathrm{BDH}$ parameter generator, the advantage $A d v_{I G}(\mathrm{~B})$ that an algorithm $\mathrm{B}$ has in solving the $\mathrm{BDH}$ problem is defined to be the probability that B outputs $e(g, g)^{x y z}$ on inputs $G_{1}, G_{2}, e, g, g^{x}, g^{y}, g^{z}$,where $\left(G_{1}, G_{2}, e\right)$ is the output of $I G$ for a sufficiently large security parameter $K, g$ is a random generator of $G_{1}$, and $x, y, z$ are random elements of $Z_{q}^{*}$. The $\mathrm{BDH}$ assumption is that $A d v_{I G}(\mathrm{~B})$ is negligible for any efficient $B$.

\section{PROXY RE-ENCRYPTION WITH PRIVATE SEARCHING}

Definition 3.1 Proxy Re-encryption with Private Searching (PRPS) scheme consists of seven randomized polynomial time algorithms as follows:

- Key Generation (KG): takes a sufficiently large security parameter $K_{1}$ as input, and produces a key pair $\left(A_{\text {pub }}, A_{\text {priv }}\right)$ for a data owner $A$, where $A_{\text {pub }}, A_{\text {priv }}$ are public key and private key respectively. We write $K G\left(K_{1}\right)=\left(A_{\text {pub }}, A_{\text {priv }}\right)$. Let $K_{2}$ be a sufficiently large security parameter, we write $K G\left(K_{2}\right)=\left(S_{p u b}, S_{\text {priv }}\right)$ for the cloud provider $S$, where $S_{\text {pub }}, S_{\text {priv }}$ are public/private key respectively. Let $K_{3}$ be a sufficiently large security parameter, we write $K G\left(K_{3}\right)=\left(U_{\text {pub }}, U_{\text {priv }}\right)$ for the data user $U$, where $U_{\text {pub }}, U_{\text {priv }}$ are public/private key respectively.

- Encryption (E): this algorithm is performed by data owner $A$ to encrypt the keyword $W_{i}\left(i \in Z^{+}\right)$ and message $m$. Correspondingly, two parts, KWEnc and EMBEnc constitutes Encryption.

1) KWEnc: is a public key encryption algorithm that takes a public key ${ }^{A_{p u b}}$ and a key word $W_{i}\left(i \in Z^{+}\right)$as inputs, and produces $W_{i}$ 's cipher text $C_{W_{i}} \in C_{w}$.We write $\operatorname{KWEnc}\left(A_{\text {pub }}, W_{i}\right)=\mathrm{C}_{W_{i}}$. 
2) EMBEnc: is a public key encryption algorithm that takes public keys $S_{p u b}, A_{p u b}$ and message $m \in M$ as inputs, and produces $m^{\prime} s$ cipher text $C_{m}$.We write $\operatorname{EMBEnc}\left(S_{\text {pub }}, A_{\text {pub }}, m\right)=C_{m}$.

- Re-Encryption Key Generation (RG): A data owner takes a public key $U_{\text {pub }}$ and private key $A_{\text {priv }}$ as inputs, and produces the re-encryption key ${ }^{r k_{A \rightarrow U}}$. We write $R G\left(A_{\text {priv }}, U_{\text {pub }}\right)=r k_{A \rightarrow U}$.

- TCompute: User takes private key $U_{\text {priv }}$ and a keyword $W_{j}\left(j \in Z^{+}\right)$as inputs, and produces $W_{j}$ 's $\operatorname{trapdoor}^{T_{W_{j}}}$. We write

$$
\operatorname{TCompute}\left(U_{\text {priv }}, W_{j}\right)=T_{W_{j}} \text {. }
$$

- Re-Encryption(R): The cloud provider takes reencryption key $r k_{A \rightarrow U}$, cipher text $C_{m}$ and some intermediate result $\theta$ as the inputs, and produces cipher text $C_{m}$ 's re-encrypted cipher text $C_{U}$. We write $\operatorname{Re}-\operatorname{Encryption}\left(\theta, r k_{A \rightarrow U}, C_{m}\right)=C_{U}$.

- Test : The cloud provider takes re-encryption key $r k_{A \rightarrow U}$, an encrypted keyword $C_{W_{i}}$ and a trapdoor $T_{W_{j}}$ as inputs, and produces " 1 " if $W_{i}=W_{j}$ or " 0 " otherwise. This algorithm is to check whether the cipher text ${ }^{C_{W_{i}}}$ matches the trapdoor $T_{W_{j}}$.

- Decryption (D): The user takes private key $U_{\text {priv }}$ and re-encrypted cipher text $C_{U}$ as inputs, and outputs the plaintext ${ }^{m}$.

Note. RG algorithm implies that the PRPS scheme is non-interactive, which means re-encryption keys can be generated by a data owner via the user's public key. No trusted third party or interaction is required.

We define security for the PRPS scheme in the sense of semantic security. Semantic security captures the intuition that given a cipher text, the adversary learns nothing about the corresponding plaintext, thus we also say that a semantically secure scheme is IND-CPA secure [9]. We first define semantic security for KWEnc and EMBEnc, and then give the definition of semantically secure PRPS scheme.

Definition 3.2 (Semantic Security of KWEnc): Given a public key encryption algorithm KWEnc which encrypts keywords using $A_{\text {pub }}$, let $\mathrm{A}_{1}$ be a polynomial time IND-CPA adversary that can adaptively ask for the trapdoor $T_{W_{i}}$ for any keyword $W_{i} \in W$ of its choice. $\mathrm{A}_{1}$ first chooses two keywords $W_{0}$ and $W_{1}$, which are not to be asked for trapdoors previously, and sends them to KWEnc. And then KWEnc picks a random element $b_{1} \in\{0,1\}$ and gives $\mathrm{A}_{1}$ the cipher text
$C_{W_{b_{1}}}=K W E n c\left(A_{p u b}, W_{b_{1}}\right)$. Finally, $\mathrm{A}_{1}$ outputs a guess $b_{1}^{\prime} \in\{0,1\}$ for $b_{1}$. We define the advantage of $A_{1}$ in breaking KWEnc as

$$
A d v_{\mathrm{A}_{1}}(k)=\left|\operatorname{Pr}\left[b_{1}=b_{1}^{\prime}\right]-\frac{1}{2}\right| \text {. }
$$

KWEnc is semantically secure if for any polynomial time $\operatorname{adversary} \mathrm{A}_{1}, A d v_{\mathrm{A}_{1}}(k)$ is negligible.

Definition 3.3 (Semantic Security of EMBEnc): Given a public key encryption algorithm EMBEnc which encrypts the message using $A_{p u b}$ and $S_{p u b}$. Let $\mathrm{A}_{2}$ be a polynomial time IND-CPA adversary that can adaptively ask for the cipher text for any message $m_{i} \in M$ of its choice. We use subscript $T$ to denote the target user, ${ }^{x}$ to denote the adversarial users, and $h$ to denote the honest users (other than $T$ ). The input marked with a ' $*$ ' is optional. $\mathrm{A}_{2}$ first chooses two messages $m_{0}$ and $m_{1}$, which are not to be asked for the cipher text previously, and sends them to EMBEnc. And then EMBEnc picks a random $b_{2}^{\prime} \in\{0,1\}$ and gives $A_{2}$ the cipher text

$$
C_{m_{b_{2}}}=\operatorname{EMBEnc}\left(A_{\text {pub }}, S_{p u b}, m_{b_{2}}\right) \text {. }
$$

Finally, $\mathrm{A}_{2}$ outputs a guess $b_{2}^{\prime} \in\{0,1\}$ for $b_{2}$. That is, for all PPT algorithms $A_{k}$,

$$
\begin{array}{r}
\operatorname{Pr}\left[\left(p k_{T}, s k_{T}\right) \leftarrow K G\left(1^{k}\right),\left\{\left(p k_{x}, s k_{x}\right) \leftarrow K G\left(1^{k}\right)\right\},\right. \\
\left\{r k_{x \rightarrow T} \leftarrow R G\left(p k_{x}, s k_{x}, p k_{T}, s k_{T}^{*}\right)\right\}, \\
\left\{\left(p k_{h}, s k_{h}\right) \leftarrow K G\left(1^{k}\right)\right\}, \\
\left\{r k_{T \rightarrow h} \leftarrow R G\left(p k_{T}, s k_{T}, p k_{h}, s k_{h}^{*}\right)\right\}, \\
\left\{r k_{h \rightarrow T} \leftarrow R G\left(p k_{h}, s k_{h}, p k_{T}, s k_{T}^{*}\right)\right\}, \\
\left(m_{0}, m_{1}, \alpha\right) \leftarrow A_{k}\left(p k_{T},\left\{\left(p k_{x}, s k_{x}\right)\right\},\left\{p k_{h}\right\},\left\{r k_{x \rightarrow T}\right\},\left\{r k_{T \rightarrow h}\right\},\left\{r k_{T \rightarrow D}\right\}\right), \\
b_{2} \leftarrow\{0,1\}, b_{2}^{\prime} \leftarrow A_{k}\left(\alpha, E M B E n c\left(p k_{T}, m_{b_{2}}\right)\right): \\
\left.b_{2}=b_{2}^{\prime}\right]<1 / 2+1 / p o l y(k)
\end{array}
$$

We define the advantage of $\mathrm{A}_{2}$ in breaking EMBEnc as

$$
A d v_{\mathrm{A}_{2}}(k)=\left|\operatorname{Pr}\left[b_{2}=b_{2}^{\prime}\right]-\frac{1}{2}\right| \text {. }
$$

We say that EMBEnc is semantically secure if for any polynomial time adversary $\mathrm{A}_{2}, A d v_{\mathrm{A}_{2}}(k)$ is negligible.

Definition 3.4 (Semantic Security of PRPS): Given an PRPS scheme consisting of KWEnc and EMBEnc, it takes a security parameter $K$ as input and runs the key generation algorithm Keygen to generate the public/ private key pairs $\left(A_{\text {pub }}, A_{\text {priv }}\right),\left(S_{\text {pub }}, S_{\text {priv }}\right)$ and $\left(U_{\text {pub }}, U_{\text {priv }}\right)$. Given an adversary A consisting of two polynomial time algorithms $\mathrm{A}_{1}$ and $\mathrm{A}_{2}, \mathrm{~A}_{1}$ initiates attacks on KWEnc and $\mathrm{A}_{2}$ initiates attacks on EMBEnc. We say that the PRPS Scheme is semantically secure if for any adversary A , $A d v_{\mathrm{A}}(k)=A d v_{\mathrm{A}_{1}}(k)+A d v_{\mathrm{A}_{2}}(k)$ is negligible. 


\section{CONSTRUCTION FOR PRPS}

We assume that the scheme is composed of the following parties, the data owner, data users, and cloud providers. To access data files shared by the data owner, data users download data files of their interest from cloud providers and then decrypt. The users are assumed to have the only access privilege of data file reading. The cloud providers are assumed to have abundant storage capacity and computation power.

In this work, cloud providers are viewed as "honest but curious", which means they follow the proposed protocol in general, but try to find out as much secret information as possible. More specifically, we assume cloud providers are more interested in file contents and user access privilege information than other secret information. Cloud providers might collude with malicious users for the purpose of harvesting file contents when it is highly beneficial. Communication channel between the data owner/users and cloud providers are assumed to be secured. Users may work independently or cooperatively. In addition, each party is preloaded with a public/private key pair and the public key can be easily obtained by other parties when necessary.

The main design goal is to help the data users achieve efficient private querying and downloading the encrypted files stored in cloud providers. The data owner won't need to re-encrypt the files in cloud provider for different users. We also want to prevent cloud providers from being able to learn both the data file contents and user queries information.

The details of construction are as follows:

Suppose data owner $A$ is about to store an encrypted file with keywords $W_{1}, \ldots W_{l}$ on a cloud storage $S$, where $l \in Z^{+}$. Keywords may be words in headline or stored date, and are relatively small. $A$ encrypts the file message using his public key ${ }^{A_{p u b}}$, the cloud storage's public key $S_{p u b}$. And then $A$ encrypts keywords $W_{1}, \ldots W_{l}$ using his public key $A_{\text {pub }}$. The file deposited in the cloud storage $S$ by the data owner $A$ is as follows:

$M S G_{U 2 S}=\left[\operatorname{EMBEnc}\left(A_{p u b}, S_{p u b}, m\right), \operatorname{KWEnc}\left(A_{p u b}, W_{1}\right), \ldots, K W E n c\left(A_{p u b}, W_{l}\right)\right]$ Where EMBEnc, KWEnc are public key encryption algorithms. Finally, $A$ appends to the encrypted file message with all the encrypted keywords and sends ${ }^{M S G_{U 2 S}}$ to $S$.

Given a sufficiently large security parameter $K \in Z^{+}$, it runs $I G$ to generate a prime $q$, two groups $G_{1}$ and $G_{2}$ of prime order $q$, and a bilinear map $e: G_{1} \times G_{1} \rightarrow G_{2}$, $g, h \in G_{1}, Z=e(g, g) \in G_{2}$, where $g$ is a generator of $G_{1}$. Then it chooses two hash functions $H_{1}, H_{3}:\{0,1\}^{*} \rightarrow G_{1}^{*}$, hash function $H_{2}: G_{2} \rightarrow\{0,1\}^{\log q}$, and hash function $H_{4}: G_{2} \rightarrow\{0,1\}^{n}$ for some ${ }^{n}$, where $H_{1}, H_{2}, H_{3}$ and $H_{4}$ are random oracles. Finally, it picks three random elements $a, b, c \in Z_{q}^{*}$ and computes $g^{a}, g^{b}$ and $g^{c}$.The plaintext space includes $M \in\{0,1\}^{n}$ and $W \in\{0,1\}^{*}$. The cipher text space includes $C_{M}=G_{1}^{*} \times\{0,1\}^{n}$ and $C_{W} \in G_{2}$.

- Key Generation (KG): The data owner $A$ 's public key is $A_{\text {pub }}=g^{a}$ with the corresponding private key $A_{\text {priv }}=a$; the user $U$ 's public/private key is $U_{p u b}=g^{b}, U_{\text {priv }}=b$ respectively. The cloud provider $S$ 's public key is $S_{p u b}=g^{c}$ with the corresponding private key $S_{\text {priv }}=c$.

- Encryption (E): This encryption algorithm consists of KWEnc and EMBEnc. The data owner first picks a random element $r \in Z_{q}^{*}$.

1) KWEnc( $\left.E_{1}\right)$ : To encrypt $m$ 's keywords $W_{1}, \ldots W_{k}\left(k \in Z^{+}\right)$under a data owner's public key $g^{a}$ and a random element $r$, it computes $H_{2}\left(e\left(g^{a}, H_{1}\left(W_{i}\right)\right)^{r}\right)$, where $W_{i} \in\left\{W_{1}, \ldots W_{k}\right\}$, sets the cipher text $C_{W_{i}}=H_{2}\left(e\left(g^{a}, H_{1}\left(W_{i}\right)\right)^{r}\right)$.

2) EMBEnc $\left(E_{2}\right)$ : To encrypt the file message $m$ under data owner's public key $g^{a}$, cloud provider's public key $g^{c}$ and random element $r$, it picks a random element $\rho \in\{0,1\}^{n}$, and computes

$$
\begin{gathered}
u_{1}=h^{r}, \\
u_{2}=\rho \oplus H_{4}\left(e\left(h^{a}, g^{c}\right)^{r}\right), \\
u_{3}=m \cdot e\left(H_{3}(\rho), g^{a}\right)^{r},
\end{gathered}
$$

and sets the cipher text $C_{m}=\left(u_{1}, u_{2}, u_{3}\right)$.

- Re-Encryption KeyGeneration (RG): Data owner $A$ delegates to user $U$ by publishing the reencryption key $r k_{A \rightarrow U}=g^{a b r}$, computed with $U$ 's public key $g^{b}$.

- Tcompute: To retrieve the file containing keyword $^{W_{j}}\left(j \in Z^{+}\right)$, user computes the trapdoor $T_{W_{j}}=H_{1}\left(W_{j}\right)^{1 / b}$ using his/her private key $U_{\text {priv }}=b$, then sends the trapdoor to the cloud provider.

- Re-Encryption( $R$ ): to change the cipher text $C_{m}=\left(u_{1}, u_{2}, u_{3}\right)$ for $A$ into a cipher text $C_{U}=\left(u_{3}, u_{4}\right)$ for $U$ under the re-encryption key $r k_{A \rightarrow U}=g^{a b r}$, it computes

$$
u_{4}=e\left(H_{3}(\rho), r k_{A \rightarrow U}\right)=e\left(H_{3}(\rho), g^{a b r}\right) .
$$

The cloud provider sends $C_{U}$ to the user.

Note. Since 
$\rho=u_{2} \oplus H_{4}\left(e\left(h^{a}, g^{c}\right)^{r}\right)=u_{2} \oplus H_{4}\left(e\left(g^{a}, h^{r}\right)^{c}\right)$,

the cloud provider can compute the intermediate value

$\rho$ with its private key ${ }^{c}$.

- Test: To determine whether a given file contains keyword $W_{j}$, the cloud provider tests whether $C_{W_{i}}=H_{2}\left(e\left(r k_{A \rightarrow U}, T_{W_{j}}\right)\right)$

If so, $\operatorname{Test}\left(r k_{A \rightarrow U}, C_{W_{i}}, T_{W_{j}}\right)$ outputs 1 , and 0 otherwise.

Note. If $W_{i}=W_{j}$, since $C_{W_{i}}=H_{2}\left(e\left(g^{a}, H_{1}\left(W_{i}\right)\right)^{r}\right)$, then $C_{W_{i}}=H_{2}\left(e\left(g^{a}, H_{1}\left(W_{j}\right)\right)^{r}\right)=H_{2}\left(e\left(g^{a b r}, H_{1}\left(W_{j}\right)^{1 / b}\right)\right)=H_{2}\left(e\left(r k_{A \rightarrow U}, T_{W_{j}}\right)\right)$

- Decryption(D) : Given the cipher text $C_{U}=\left(u_{3}, u_{4}\right) \quad$ it computes $m=u_{3} /\left(u_{4}\right)^{\frac{1}{U_{\text {priv }}}}=u_{3} /\left(u_{4}\right)^{\frac{1}{b}}$ to recover the message $m$.

Note that:

$$
\frac{u_{3}}{\left(u_{4}\right)^{\frac{1}{b}}}=\frac{m \cdot e\left(H_{3}(\rho), g^{a}\right)^{r}}{\left(e\left(H_{3}(\rho), g^{a b}\right)^{r}\right)^{\frac{1}{b}}}=\frac{m \cdot e\left(H_{3}(\rho), g^{a}\right)^{r}}{e\left(H_{3}(\rho), g^{a}\right)^{r}}=m
$$

\section{ANALYSIS}

\section{A. Security Analysis}

\section{1) Privacy for Keyword}

Lemma 5.1 (Privacy for Keyword) Let $H_{1}$ be a random oracle from $\{0,1\}^{*}$ to $G_{1}^{*}$ and $H_{2}$ be a random oracle from $G_{2}$ to ${ }^{\{0,1\}^{\log q}}$. Suppose $\mathrm{A}_{1}$ be an IND-CPA adversary that has the advantage ${ }^{\varepsilon_{1}}$ in breaking KWEnc. Suppose $\mathrm{A}_{1}$ makes at most $q_{H_{2}}>0$ hash queries to $H_{2}$ and at most $q_{T}>0$ trapdoor queries. Then there is an algorithm $B_{1}$ that solves the $\mathrm{BDH}$ problem with the advantage at least $\varepsilon_{1}^{\prime}=2 \varepsilon_{1} /\left\{e \cdot q_{H_{2}} \cdot\left(1+q_{T}\right)\right\}$, and a running time $O\left(\right.$ time $\left.\left(\mathrm{A}_{1}\right)\right)$.

Proof. The proof is similar to Lemma 4.2 in [6].

Privacy for Keyword guarantees the user's query privacy, namely, the cloud provider learns nothing about what the user's querying for in this process. In our scheme, the file is encrypted with the data owner's public key before its storage in the untrusted cloud. A user sends a trapdoor with inputting encrypted keyword to query for a file which including the encrypted keyword. The cloud provider will have no knowledge of the file's keyword, only if it obtains the private key of the data owner.

2) Privacy for Message

Our security definition quantifies over all encryption algorithms; in this case, we have two algorithms EMBEnc $\left({ }^{E_{2}}\right)$ and Re-Encryption $(R)$, where an $E_{2}$ cipher text takes the form $u_{3}=m \cdot e\left(H_{3}(\rho), g^{a}\right)^{r}$. This construction is equivalent to $R$ cipher text of the form $_{4} u_{4}=e\left(H_{3}(\rho), g^{a r}\right)^{b}$. Now, it is clear if the $E_{2}$ cipher text of the form $u_{3}=m \cdot e\left(H_{3}(\rho), g^{a}\right)^{r}$ is secure, then so is the $R$ version, since $E_{2}$ cipher texts reveal more information. Thus, it suffices to argue the security of the $E_{2}$ cipher texts only.

Next, we show that EMBEnc is a semantically secure public key encryption if the $\mathrm{BDH}$ assumption holds. It is worth noticing that the outer attackers couldn't calculate $\rho$ if the $\mathrm{BDH}$ assumption holds. Without loss of generality, we suppose that an IND-CPA adversary $\mathrm{A}_{2}$ has already known $\rho$ and could issue ${ }^{H_{3}}$ queries at any time.

Lemma 5.2 (Privacy for Message) Let ${ }^{H_{3}}$ be a random oracle from $\{0,1\}^{*}$ to $G_{1}^{*}$ and $H_{4}$ be a random oracle from $G_{2}$ to $\{0,1\}^{n}$. Let $\mathrm{A}_{2}$ be an IND-CPA adversary that has the advantage $\varepsilon_{2}$ against EMBEnc. Suppose $\mathrm{A}_{2}$ makes $q_{H_{3}}>0$ hash function queries to $H_{3}$ and $q_{R}>0$ queries to Request . Then there is an algorithm $B_{2}$ that solves the $\mathrm{BDH}$ problem with the advantage at least $\varepsilon_{2}^{\prime}=2 \varepsilon_{2} / q_{\mathrm{H}_{3}} q_{R}$ and a running time $\mathrm{O}\left(\operatorname{time}\left(\mathrm{A}_{2}\right)\right)$.

Proof . See Appendix A.

3) Security for PRPS

We will study the security for our PRPS scheme according to Definition 3.4. The following theorem shows that PRPS is semantically secure if the $\mathrm{BDH}$ problem is hard.

Theorem 5.1 (Security for PRPS). Suppose the hash functions $\mathrm{H}_{1}, \mathrm{H}_{2}, \mathrm{H}_{3}$ and $\mathrm{H}_{4}$ are random oracles. Let ${ }^{\mathrm{A}}$ be an IND-CPA adversary consisting of two polynomial time algorithms $A_{1}$ and $A_{2}$. Let $A_{1}$ be an IND-CPA adversary that has the advantage ${ }^{\varepsilon_{1}}$ in breaking KWEnc. Suppose $\mathrm{A}_{1}$ makes $q_{T}>0$ trapdoor queries and $q_{\mathrm{H}_{2}}>0$ hash queries to $\mathrm{H}_{2}$. Let $\mathrm{A}_{2}$ be an IND-CPA adversary that has the advantage ${ }^{\varepsilon_{2}}$ against EMBEnc. Suppose $\mathrm{A}_{2}$ makes $q_{\mathrm{H}_{3}}>0$ hash function queries to $H_{3}$ and $q_{R}>0$ queries to Request . Let $\mathrm{A}$ be an IND-CPA adversary that has the advantage $\varepsilon=\varepsilon_{1}+\varepsilon_{2}$ against the PRPS scheme. Then there is an algorithm $\mathrm{B}$ that solves the $\mathrm{BDH}$ problem with the advantage at least: $A d v_{\mathrm{B}} \geq 2 \varepsilon_{1} /\left\{e \cdot q_{\mathrm{H}_{2}} \cdot\left(1+q_{T}\right)\right\}+2 \varepsilon_{2} / q_{H_{3}} q_{R}$ That means the PRPS scheme is semantically secure under the BDH problem. Here $e \approx 2.71$ is the base of the natural logarithm. The running time of $\mathrm{B}$ is $O(\operatorname{time}(\mathrm{A}))$.

Proof. PRPS includes two public key encryption algorithms, i.e. EMBEnc and KWEnc. Therefore, the proof follows directly from Lemma 5.1 and Lemma 5.2. 


\section{B. Efficiency Analysis}

This section evaluates the efficiency of the PRPS scheme in terms of the computation overhead introduced by each operation. We use computation time to denote the computation overhead of the algorithm operated by different roles (for example, the data owner, the user). Encryption (KWEnc, EMBEnc) and Re-Encryption Key Generation are operated by the data owner; ReEncryption and Test are operated by the cloud provider and the user's operation are Tcompute and Decryption.

Suppose the runtime of exponent arithmetic (EXP) is $T_{e}$, the runtime of hash arithmetic (Hash) is $T_{h}$ and the runtime of arithmetic of bilinear pairings (Pairing) is $T_{b}$.

TABLE I.

COMPUTATION EFFICIENCY OF PRPS

\begin{tabular}{|l|l|l|l|l|l|}
\hline \multicolumn{2}{|c|}{} & EXP & Hash & Pairing & Total \\
\hline \multirow{2}{*}{$\begin{array}{l}\text { Data } \\
\text { owner }\end{array}$} & Encryption & $5 T_{e}$ & $4 T_{h}$ & $3 T_{b}$ & \multirow{2}{*}{$6 T_{e}+4 T_{h}+3 T_{b}$} \\
\cline { 2 - 5 } & $\begin{array}{l}\text { Re-Encryption } \\
\text { KeyGeneration }\end{array}$ & $T_{e}$ & & & \\
\hline $\begin{array}{l}\text { Cloud } \\
\text { Provid } \\
\text { er }\end{array}$ & Re-Encryption & $2 T_{e}$ & $2 T_{h}$ & $2 T_{b}$ & \multirow{2}{*}{$2 T_{e}+3 T_{h}+3 T_{b}$} \\
\cline { 2 - 5 } & Test & & $T_{h}$ & $T_{b}$ & \\
\hline \multirow{2}{*}{ User } & Tcompute & & $T_{h}$ & & \multirow{2}{*}{$T_{e}+T_{h}$} \\
\cline { 2 - 5 } & Decryption & $T_{e}$ & & & \\
\hline
\end{tabular}

The comparison in the runtimes for the cryptographic operations in PRPS scheme is given in TABLE I. These results indicate that the runtimes of hash arithmetic and exponent arithmetic operated by a user are much less than the ones of cloud provider's and data owner's operations. The scheme transfers most computation cost from the user to the cloud provider decrease the computation overhead and enhance the efficiency of the user. That makes sense to the application of cloud computing with thin clients.

Note. In our scheme, a data owner takes his own private key, the user's public key and a random element as the inputs, and produces re-encryption key $r_{A \rightarrow U}=\left(g^{b}\right)^{a r}=g^{a b r}$. Thus, there is no need to deliver the user's private key to the data owner or interact with the third party for the re-encryption key, which implies that our PRPS scheme is non-interactive.

\section{CONCLUSIONS}

In this paper, we propose an efficient proxy reencryption with private searching (PRPS) scheme in the untrusted cloud. We exploit proxy re-encryption and uniquely combining it with techniques of public key encryption with keyword search and dual receiver cryptosystem. PRPS allows users and data owners to query and access files storage in untrusted cloud provider, while maintaining query privacy and data privacy. It allows user to decrypt the files efficiently. The PRPS scheme is proven semantically secure in the random oracle model. We indicate that the challenging "Private Searching on Encrypted Data" problem is of independent interest and deserved further study.

\section{APPENDIX A PROOF OF LEMMA 5.2}

Proof. ${ }^{B_{2}}$ Is given $\rho \in\{0,1\}^{n}, \mu_{0}=g, \mu_{1}=g^{\alpha_{2}}$, $\mu_{2}=g^{\beta_{2}}, \quad \mu_{3}=g^{\gamma_{2}} \in G_{1}$, where $\alpha_{2}, \beta_{2}, \gamma_{2}$ are random elements in $Z_{q}^{*}$. Its goal is to output $D_{2}=e(g, g)^{\alpha_{2} \beta_{2} \gamma_{2}} \in G_{2}$. $\mathrm{B}_{2}$ finds $\mathrm{D}_{2}$ by interacting with $\mathrm{A}_{2}$ as follows:

Keygen: ${ }^{\mathrm{B}_{2}}$ sends $\left(\mu_{0}, \mu_{1}\right)$ as the public key to $\mathrm{A}_{2}$.

$H_{3}$-Queries : $\mathrm{B}_{2}$ maintains a list of tuples called $H_{3}-L i s t$, in which each entry is a tuple of the form $\left\langle\rho_{j}, f_{j}\right\rangle$. The list is initially empty. When $\mathrm{A}_{2}$ issues a query to ${ }_{3}, B_{2}$ checks if $\rho_{i}$ is already on $H_{3}-$ List in the form of $\left\langle\rho_{j}, f_{j}\right\rangle$. If so $\mathrm{B}_{2}$ responds to $\mathrm{A}_{2}$ with $H_{3}\left(\rho_{i}\right)=f_{i}$. Otherwise, $\mathrm{B}_{2}$ picks a random $d \in Z_{q}^{*}$, computes $f_{i}=\mu_{2} \cdot g^{d}=g^{\beta_{2}} \cdot g^{d} \in G_{1}^{*}$ adds the tuple $\left\langle\rho_{i}, f_{i}\right\rangle$ to $H_{3}-$ List , and responds to $\mathrm{A}_{2}$ with $H_{3}\left(\rho_{i}\right)=f_{i}$.

$H_{4}$-Queries : $\mathrm{B}_{2}$ maintains a list of tuples called $H_{4}-L i s t$, in which each entry is a tuple of the form $\left\langle r_{j}, l_{j}\right\rangle$. The list is initially empty. When $\mathrm{A}_{2}$ issues a query to $H_{4}, \mathrm{~B}_{2}$ checks if $r_{i}$ is already on $H_{4}-$ List in the form of $\left\langle r_{i}, l_{i}\right\rangle$. If so, $\mathrm{B}_{2}$ responds to $\mathrm{A}_{2}$ with $H_{4}\left(r_{i}\right)=l_{i}$. Otherwise, ${ }^{B_{2}}$ picks a random string ${ }{ }_{i} \in\{0,1\}^{n}$, adds the tuple $\left\langle r_{i}, l_{i}\right\rangle$ to $H_{4}-$ List , and responds to $\mathrm{A}_{2}$ with $H_{4}\left(r_{i}\right)=l_{i}$.

Request : Next, for $i=1$ up to $\operatorname{poly}(k),{ }_{2}$ can request:

a. ${ }^{r k_{x \rightarrow T}}$, a delegation to $T$ from a party corrupted by $A_{2}$. $A_{2}$ can generate these delegations for as many corrupted users as it likes internally by running $\left(p k_{x}, s k_{x}\right) \leftarrow K G\left(1^{k}\right)$ and computing $r k_{x \rightarrow T}=\left(g^{\alpha_{2}}\right)^{s k_{x}}$.

b. $r k_{T \rightarrow h}$, a delegation from $T$ to an honest party $h$. The simulator randomly selects one values $r_{h} \leftarrow Z_{q}$, sets $r k_{T \rightarrow h}=\left(\mu_{0}\right)^{r_{h}}=g^{r_{h}}$ And $p k_{h}=g^{r_{h}}$, and sends $\left(p k_{h}, r k_{T \rightarrow h}\right)$ to $A_{2}$. The corresponding secret key is $s k_{h}=r_{h}$.

c. ${ }^{r k_{h \rightarrow T}}$, a delegation to $T$ from an honest party $h$.

The simulator uses either the recorded value ${ }^{r_{h}}$ from the previous step if the honest party already exists, or generates fresh random values for a new party, and computes $r k_{h \rightarrow T}=\left(g^{\alpha_{2}}\right)^{r_{h}}$.

Challenge. $\mathrm{A}_{2}$ outputs two messages $m_{0}$ and $m_{1}$ on which it wishes to be challenged. ${ }^{B_{2}}$ randomly picks $b_{2} \in\{0,1\}$ and a random string $S_{2} \in\{0,1\}^{n}$, and gives the cipher text $C_{2}=\left(\mu_{3}, S_{2}\right)$ to $\mathrm{A}_{2}$. Note that the decryption of the cipher text is: 


$$
\begin{aligned}
\mu_{3} & =m \cdot\left(e\left(H_{3}(\rho), \mu_{1}\right)^{\gamma_{2}}\right)=m \cdot\left(e\left(H_{3}(\rho), g^{\alpha_{2}}\right)^{\gamma_{2}}\right) \\
& =m \cdot\left(e\left(g^{\beta_{2}} \cdot g^{d}, g^{\alpha_{2}}\right)^{\gamma_{2}}\right)=m \cdot\left(e(g, g)^{\alpha_{2} \gamma_{2}\left(\beta_{2}+d\right)}\right)
\end{aligned}
$$

Hence, $C_{2}$ is a valid cipher text for $m_{b_{2}}$ as required.

Guess: ${ }^{A_{2}}$ outputs its guess $b_{2}^{\prime} \in\{0,1\}$ for $b_{2}, B_{2}$ picks a random pair $\left\langle\rho_{j}, f_{j}\right\rangle$ from $H_{3}-$ List and outputs $f_{j}$ as the solution to the given instance of $\mathrm{BDH}$.

Let $Q_{2}$ be the event that $\mathrm{A}_{2}$ issues a query for $f$. From proof of Lemma 5.1, we know that $\operatorname{Pr}\left[Q_{2}\right] \geq 2 \varepsilon_{2}$. That means $\mathrm{A}_{2}$ will issue a query for $f$ with the probability at least $2 \varepsilon_{2}$ B $_{2}$ will choose the correct pair with the probability at least $1 / q_{H_{3}}$ and succeed in Request with the probability at least $1 / q_{R}$, thus ${ }^{B_{2}}$ produces the correct answer with the probability at least $\varepsilon_{2}^{\prime}=2 \varepsilon_{2} / q_{H_{3}} q_{R}$ as required. If $\mathrm{A}_{2}$ has the advantage $\varepsilon_{2}$ against EMBEnc, then $\mathrm{B}_{2}$ succeeds with probability $\varepsilon_{2}^{\prime}=2 \varepsilon_{2} / q_{H_{3}} q_{R}$.This contradicts the BDH assumption.

\section{ACKNOWLEDGMENT}

This work is partially supported by National High Technology Research and Development Program of China (863 Program) under Grant No. 2009AA01Z423 and the Fundamental Research Funds for the Central Universities under Grant No. 2009JBM004.

\section{REFERENCES}

[1] M. Armbrust, A. Fox, R. Griffith, A. D. Joseph, R. H. Katz, A. Konwinski, G. Lee, D. A. Patterson, A. Rabkin, I. Stoica, and M. Zaharia, “A View of Cloud Computing," Communications of the ACM, Vol. 53, pp.50-58,April 2010.

[2] Shucheng Yu, Cong Wang, Kui Ren and Wenjing Lou, "Achieving Secure, Scalable, and Fine-grained Data Access Control in Cloud Computing," in Proceedings of INFOCOM 2010. IEEE, 2010.

[3] G. Ateniese, K. Fu, M. Green, S. Hohenberger, "Improved proxy re-encryption schemes with applications to secure distributed storage," ACM Transactions on Information and System Security (TISSEC). 9 (1) (2006) 1-30.

[4] D. Boneh, G. Crescenzo, R. Ostrovsky, and G. Persiano. "Public Key Encryption with Keyword Search," Proceedings of Eurocrypt 2004, Lecture Notes in Computer Science 3027, Springer-Verlag. 2004. pp. 506522.

[5] T. Diament, H. K. Lee, A. D. Keromytis and M. Yung, "The Dual Receiver Cryptosystem and its Application," Proceedings of the ACM CCS 2004, pp. 330-343.

[6] Qin Liu, Guojun Wang, Jie Wu, “An Efficient Privacy Preserving Keyword Search Scheme in Cloud Computing,"in Computational Scinece and Engineering, CSE'09, vol.2, 2009 , pp. 715 - 720.

[7] J. Shao, Z. Cao, X. Liang, H. Lin, "Proxy re-encryption with keyword search," Information Science.vol.180, pp. 2576-2587, 2010.

[8] R. Canetti, S. Hohenberger, "Chosen-cipher text secure proxy re-encryption,” in: ACM CCS 2007, 2007. Full version: Cryptology ePrint Archieve: Report 2007/171.
[9] D. Boneh and M. Franklin. "Identity Based Encryption from the Weil Pairing," SIAM J. of Computing, 32 (3): 586-615, 2003.

[10] D. Song, D. Wagner, A. Perrig, "Practical techniques for searching on encrypted data". IEEE Symp. On Research in Security and Privacy 2000, IEEE. 2000. pp.44-55

[11] Y. Chang, M. Mitzenmacher, "Privacy preserving keyword searches on remote encrypted data". Proceedings of ACNS2005. Lecture Notes in Computer Science 3531, Springer-Verlag. 2005. pp.442-455.

[12] Melissa Chase, Seny Kamara, "Structured Encryption and Controlled Disclosure”. Proceedings of ASIACRYPT 2010, Lecture Notes in Computer Science 6477, Springer-Verlag. 2010. pp. 577-594.

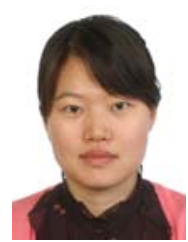

Xi Chen is currently pursuing the M.S degree with Department of Information and Communication Engineering in Beijing Jiaotong University. Her research interests include cryptographic protocols and information security.

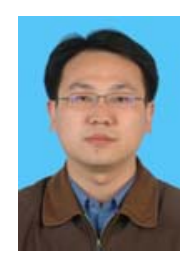

Yong Li received his M.S. degree in Computer Science from Wuhan University in 2003, and the Ph.D. degree from State Key Laboratory of Information Security, Graduate University of Chinese Academy of Sciences in 2007. School of Electronic and Information Engineering, Beijing Jiaotong University. He has over 20 publications and filed 6 patents. His research interests include cryptographic protocols and information security.

Dr. Li is a member of the International Association for Cryptologic Research (IACR), Chinese Association for Cryptologic Research (CACR), Association for Computing Machinery (ACM), China Computer Federation (CCF). He served as organizing committee chair of the international conference on cloud computing (CloudCom 2009). He was the program committee member for the international conferences CloudCom 2009, CloudCom 2010, NCIS'11. He also served as peer reviewers for seveal international conferences and academic journals, such as Asiacrypt, PKC, FSE, ACNS, Journal of Systems and Software, High Technology Letters, etc. 